\title{
ARTICLE
}

Cellular and Molecular Biology

\section{Acetyl-bufalin shows potent efficacy against non-small-cell lung cancer by targeting the CDK9/STAT3 signalling pathway}

\author{
Lehe Yang ${ }^{1,2}$, Feng Zhou ${ }^{1,2}$, Yan Zhuang ${ }^{1,3}$, Yanan Liu ${ }^{1,3}$, Lingyuan Xu ${ }^{1,2}$, Haiyang Zhao ${ }^{3}$, Youqun Xiang ${ }^{2}$, Xuanxuan Dai ${ }^{2}$, Zhiguo Liu $^{1,2}$,
} Xiaoying Huang ${ }^{2}$, Liangxing Wang ${ }^{2}$ and Chengguang Zhao $\mathbb{D}^{1,2}$

BACKGROUND: Cyclin-dependent kinase 9 (CDK9) is a promising prognostic marker and therapeutic target in cancers. Bufalin is an effective anti-tumour agent; however, the clinical application of bufalin is limited due to its high toxicity. Acetyl-bufalin, the bufalin prodrug, was designed and synthesised with higher efficiency and lower toxicity.

METHODS: Three non-small-cell lung cancer (NSCLC) cell lines, a xenograft model and a patient-derived xenograft (PDX) model were used to examine the effects of acetyl-bufalin. CDK9/STAT3 involvement was investigated by knockdown with siRNA, proteome microarray assay, western blot analysis and co-immunoprecipitation experiments. Acute toxicity test and pharmacokinetics (PK) study were conducted to assess the safety and PK. The human NSCLC tissues were analysed to verify high CDK9 expression. RESULTS: We showed that CDK9 induced NSCLC cell proliferation and that this effect was associated with STAT3 activation, specifically an increase in STAT3 phosphorylation and transcription factor activity. Acetyl-bufalin is an effective and safety inhibitor of the CDK9/STAT3 pathway, leading to the impediment of various oncogenic processes in NSCLC. Molecular docking and highthroughput proteomics platform analysis uncovered acetyl-bufalin directly binds to CDK9. Consequently, acetyl-bufalin impaired the complex formation of CDK9 and STAT3, decreased the expressions of P-STAT3, and transcribed target genes such as cyclin B1, CDC2, MCL-1, Survivin, VEGF, BCL2, and it upregulated the expression levels of BAX and caspase-3 activity. Acetyl-bufalin inhibited tumour growth in NSCLC xenograft and PDX models.

CONCLUSIONS: Acetyl-bufalin is a novel blocker of the CDK9/STAT3 pathway thus may have potential in therapy of NSCLC and other cancers.

British Journal of Cancer (2021) 124:645-657; https://doi.org/10.1038/s41416-020-01135-6

\section{BACKGROUND}

Chansu, the dried skin secretions of the giant toads, Bufo bufo gargarizans Cantor and Bufo melanostictus Schneider, is an important traditional Chinese medicine that is widely used in China and other Asian countries to clinically treat several ailments. ${ }^{1}$ Cinobufacini (Huachansu) is a water-soluble extract from the skins and parotid venom glands of the toad, Bufo bufo gargarizans Cantor, which has been approved by the Chinese State Food and Drug Administration (SFDA; IS09002). ${ }^{2,3}$ Cinobufacini is widely used to treat patients with liver, lung, colon and pancreatic cancers at oncology clinics in China. ${ }^{4-6}$ However, the pharmacological constituents and precise mechanisms of cinobufacini require further study, and its active compounds remain unclear. ${ }^{7}$ We screened the effective anti-tumour properties of 13 ingredients from toad skin on non-small-cell lung cancer (NSCLC) cells and found that bufalin was the most effective compound against NSCLC cell proliferation (Supplementary Table 1).

Bufalin is the prominent active constituent of chansu and cinobufacini. ${ }^{8,9}$ Pharmacological studies have shown that bufalin possesses significant anti-tumour effects. ${ }^{10,11}$ Although bufalin display perfectly capable of killing various tumour cells in vitro, however, it is always obtained unsatisfactory results after administration in vivo, indicating the obvious flaw in the drugability of bufalin. ${ }^{12,13}$ Because of its fast metabolism, toxicity, insolubility in water and short half-life, its application in clinics is limited. ${ }^{14}$ However, studies have shown that the substituent group could influence the bufalin metabolic enzyme interactions; thereafter, the kinetic behaviour and parameters were varied greatly. ${ }^{15}$ In this study, the bufalin prodrug, acetyl-bufalin, was designed and synthesised base on aspirin (acetylsalicylic acid) and diacerein (diacetoxyrhein) to develop new analogues with higher efficiency and lower toxicity (Supplementary Fig. S1). Acetylbufalin can help to optimise drug delivery and is expected to improve pharmacokinetics and anti-tumour activity.

Cyclin-dependent kinase 9 (CDK9) is a key transcriptional regulator and promising prognostic marker and therapeutic target in cancers. ${ }^{16,17}$ Unlike other CDKs, CDK9 does not regulate the cell cycle but promotes RNA synthesis in the genetic processes of cell growth, differentiation and viral pathogenesis. ${ }^{18-20}$ Previous data indicated that the IL6-inducible complex of CDK9/STAT3 is essential for inducing $\gamma$-fibrinogen during the hepatic acutephase response. ${ }^{21}$ Therefore, using CDK9 inhibitors to disrupt IL6/ CDK9/STAT3 signalling could be a potential therapeutic strategy for treating inflammation and cancer. In this study, IL6 strongly

\footnotetext{
${ }^{1}$ School of Pharmaceutical Sciences, Wenzhou Medical University, 325035 Wenzhou, Zhejiang, China; ${ }^{2}$ The First Affiliated Hospital, Wenzhou Medical University, 325000 Wenzhou, Zhejiang, China and ${ }^{3}$ The Institute of Life Sciences, Wenzhou University, 325035 Wenzhou, Zhejiang, China

Correspondence: Xiaoying Huang (zjwzhxy@126.com) or Liangxing Wang (wzyxywlx@163.com) or Chengguang Zhao (zhaochengguang@wmu.edu.cn)
}

Received: 17 June 2020 Revised: 4 September 2020 Accepted: 8 October 2020

Published online: 30 October 2020 
induced the CDK9 and STAT3 complex in NSCLC cells. Acetylbufalin abolished IL6-inducible P-STAT3. Moreover, acetyl-bufalin inhibited CDK9 expression and decreased phospho-RNA Pol II Ser2 in vitro and in vivo. Here, we demonstrate that acetyl-bufalin is a novel inhibitor of the CDK9/STAT3 axis.

\section{METHODS}

\section{Patient tissues}

With the approval and informed consent of the Ethics Committee of the First Affiliated Hospital of Wenzhou Medical University, a total of 12 pair human NSCLC tissues and their adjacent tissues were collected from the First Affiliated Hospital of Wenzhou Medical University. Fresh tissues were immediately snap-frozen and stored at $-80^{\circ} \mathrm{C}$ or fixed and embedded in paraffin (Supplementary Table 2).

\section{Cell culture}

Human NSCLC cell lines PC-9, H460 and A549 were purchased from the Shanghai Institute of Biosciences and Cell Resources Center (Chinese Academy of Sciences, Shanghai, China). All cells were cultured in Roswell Park Memorial Institute (RPMI)-1640 medium (Thermo Fisher Scientific) with $10 \%$ foetal bovine serum (FBS; Thermo Fisher Scientific). The cells were cultured in a humidified cell incubator at $37^{\circ} \mathrm{C}$ with $5 \% \mathrm{CO}_{2}$.

\section{Antibodies and reagents}

The antibodies against P-CDK9, CDK9, P-STAT3, BAX, S2 RNAPII and RNAPII were purchased from Abcam Co. (Cambridge, UK); The antibodies against STAT3, MCL-1, VEGF, Survivin, Cyclin B1, CDC2 and GAPDH were purchased from Cell Signal Technology (Danvers, MA, USA). The antibodies against BCL2, horseradish peroxidase (HRP)-conjugated donkey anti-rabbit lgG and HRPconjugated goat anti-mouse IgG were purchased from Santa Cruz Biotechnology Inc. (Dallas, TX, USA). Dimethyl sulfoxide (DMSO) and methylthiazolyldiphenyl-tetrazolium bromide (MTT) were purchased from Sigma-Aldrich Co. (St Louis, MO, USA). A protease phosphatase-inhibitor mixture was obtained from Applygen Technologies (Beijing, China). The caspase-3 colorimetric assay kit was purchased from Abcam Co. (Cambridge, UK). The Annexin V-FITC apoptosis Detection Kit I and propidium iodide (PI) were purchased from BD Pharmingen (Franklin Lakes, NJ, USA). A Bradford protein-assay kit, enhanced chemiluminescence kit and polyvinylidene fluoride (PVDF) membrane were obtained from Bio-Rad Laboratories (Hercules, CA, USA). Coomassie Brilliant Blue, acrylamide (30\%), tetramethylethylenediamine, Tris-glycine, sodium dodecyl sulfate, pre-stained protein marker and non-fat dry milk were from obtained Bio-Rad Laboratories. Bufalin was purchased from Herbest Biological Technology Co. (Baoji, China). SNS-032 was purchased from Selleck Chemicals (Houston, USA). The compounds used in vitro were dissolved in DMSO.

MTT cytotoxicity assay

The cytotoxicity and viability of human NSCLC cells and normal cells were determined by MTT assay. MTT assay was performed as described previously. ${ }^{22}$ MTT assay was used to determine the cell cytotoxicity and viability in human NSCLC cells. Cells $\left(5 \times 10^{3}\right.$ cells/well) were plated in 96-well plates and attached overnight. After the proper treatment, the MTT solution was added at 25 $\mu \mathrm{L} /$ well and incubated at $37^{\circ} \mathrm{C}$ for $4 \mathrm{~h}$. The formazan crystals were dissolved in $150 \mu \mathrm{L}$ of DMSO, and the optical density (OD) was measured using a Microplate Reader at $490 \mathrm{~nm}$. The cell viability was calculated by the following formula: viability $=$ (average OD values of treatment wells/average OD values of vehicle control wells) $\times 100 \%$. And half-maximal inhibitory concentration $\left(\mathrm{IC}_{50}\right)$ values were determined by GraphPad Pro Prism 7.0.
Colony-formation assay

The human NSCLC cells and normal cells were seeded at 1000 cells/well on six-well plates, placed in $5 \% \mathrm{CO}_{2}$ atmosphere and cultured overnight at $37^{\circ} \mathrm{C}$. DMSO (Control) and acetyl-bufalin (50, $100,200 \mathrm{nM}$ ) were added to the cells for $24 \mathrm{~h}$. The fresh medium was replaced every 2 days to maintain the growth of cells for 7 days. Colonies were washed with phosphate-buffered saline (PBS), fixed with $4 \%$ paraformaldehyde at room temperature for $15 \mathrm{~min}$, washed with purified water for three times and stained with Crystal violet for $10 \mathrm{~min}$. Each experiment was in triplicate, and three independent experiments were carried out.

Wound-healing migration assay

The ability of cell migration was evaluated by wound-healing assay. The cells grew to $80-90 \%$ confluence in six-well plates and then scraped the cell monolayer at the centre of the wells with the tip of a sterile $10 \mu \mathrm{L}$ pipette to form a clean, straight wound area. Then, the wells were washed with PBS to remove detached cells from the plates. After that, cells were cultured in serum-free RPMI medium with vehicle control or acetyl-bufalin $(25,50,100 \mathrm{nM})$. The migration of cells to the wound area was photographed at the 0 , 24 and $48 \mathrm{~h}$ timepoints with a microscope and imaging system (Leica, Wetzlar, Germany).

\section{Acute toxicity test}

In the acute toxicity test, $30 \mathrm{NIH}$ mice (female, 8 weeks old) were randomly divided into three groups $(n=10)$, including vehicle group, bufalin group $(30 \mathrm{mg} / \mathrm{kg}$ ) and acetyl-bufalin group $(30 \mathrm{mg} /$ $\mathrm{kg}$ ). The three groups received intraperitoneal injection only on the first day. All the mice were housed under $12 \mathrm{~h}$ light-dark cycles at $25^{\circ} \mathrm{C}$ and free for water and diet. In addition, the mortality and weight of the mice were observed for 14 days. All the mice were sacrificed by cervical dislocation.

\section{Pharmacokinetic study}

For the pharmacokinetic study, female S.D. rats (body weight $200 \pm 10 \mathrm{~g}$ ) have fasted overnight with free access to water before the experiment. Twelve rats were randomly divided into four groups. The time schedule included eight timepoints, and three S.D. rats were sampled at each time-point. Bufalin and acetyl-bufalin were administered intravenously and intraperitoneally to the rats at the dose of $0.25 \mathrm{mg} / \mathrm{kg}$ and $2 \mathrm{mg} / \mathrm{kg}$, respectively. The animals had free access to water during the experiment. Blood samples were collected from the retro-orbital plexus of each rat at $0.083,0.25,0.5,1,2,4,6,8,12,24 \mathrm{~h}$ after administration. Plasma was separated from the blood after centrifugation at $6000 \times \mathrm{g}$ for $10 \mathrm{~min}$ and frozen at $-20^{\circ} \mathrm{C}$ until analysis. In total, $20 \mu \mathrm{L}$ of standard samples in duplicate, quality control samples in duplicate and rat plasma samples were mixed with $60 \mu \mathrm{L}$ of ethyl acetate containing IS $(200 \mathrm{ng} / \mathrm{mL}$ of tolbutamide, $50 \mathrm{ng} / \mathrm{mL}$ of propranolol and $500 \mathrm{ng} / \mathrm{mL}$ of Dic) in EP tubes. After the mixture was vortexed for $1 \mathrm{~min}$, then centrifuged for $10 \mathrm{~min}\left(13,000 \mathrm{rpm}, 4^{\circ} \mathrm{C}\right)$, transferred $50 \mu \mathrm{L}$ supernatant to a 96-well plate with $150 \mu \mathrm{L}$ purred water, shacked for $10 \mathrm{~min}$ and finally injected $5 \mu \mathrm{L}$ into liquid chromatographic-tandem mass spectrometry (LC-MS/MS) system for analysis.

\section{Cell apoptosis assay}

Cell apoptosis assay was performed as described previously. ${ }^{23}$ The human NSCLC cells and HUVEC cells were inoculated in six-well plates, cultured in complete medium to grow to $80 \%$ confluency, then treated with DMSO (Control) and acetyl-bufalin (50, 100, $200 \mathrm{nM}$ ) for $24 \mathrm{~h}$ to evaluate the effects of acetyl-bufalin on cell apoptosis. Cells were collected, washed twice in ice-cold PBS and then resuspended in the binding buffer according to the instructions of the apoptosis Kit. The treated cells (as described above) were simultaneously incubated with fluorescein-labelled 
Annexin V and PI. Annexin V-binding buffer was then added to the mixture, and fluorescence was measured on a FACSCalibur (BD Biosciences; Baltimore, MD, USA). Flowjo software was used to analyse the data.

\section{Cell cycle assay}

The PC-9 cells were seeded into six-well plates for $12 \mathrm{~h}$, and then treated with DMSO (Control) or acetyl-bufalin (50, 100, $200 \mathrm{nM})$ for $24 \mathrm{~h}$. Cells were then labelled with $\mathrm{Pl}$, and the cell cycle was analysed on a FACSCalibur. Flowjo software was used to analyse the data.

Hoechst 33342 staining

A Hoechst 33342 assay kit (Beyotime Institute of Biotechnology, China) was used to detect apoptosis in H460 and PC-9 cells. Cells were inoculated in six-well plates and incubated with DMSO (Control) and acetyl-bufalin (50, 100, $200 \mathrm{nM})$. After $24 \mathrm{~h}$, cells were washed in PBS and fixed in freshly prepared 4\% paraformaldehyde for $15 \mathrm{~min}$. Then the cells were washed with PBS again, incubated with Hoechst 33342 staining solution for $20 \mathrm{~min}$, and washed with PBS before anti-fade mounting medium was added. Apoptotic cells were detected using a fluorescence microscope (Leica, Wetzlar, Germany).

\section{Western blot analysis}

Tissue proteins were extracted with tissue protein lysate buffer. Cells were grown to different densities and extracted with cell protein lysate buffer. The protein was mixed with loading buffer and boiled for $10 \mathrm{~min}$ at $100^{\circ} \mathrm{C}$, then loaded and separated by $10 \%$ or $12 \%$ sodium dodecyl sulfate-polyacrylamide gel (SDSPAGE) and then transferred onto a PVDF membrane blocking with $5 \%$ skim milk. The blots were incubated overnight with specific primary antibodies at $4{ }^{\circ} \mathrm{C}$. On the second day, after washing with TBST (a mixture of tris-buffered saline (TBS) and Tween 20) three times, the nitrocellulose membranes were incubated with horseradish peroxidase-conjugated secondary antibodies for $1 \mathrm{~h}$. The immunoreactive bands were visualised with enhanced chemiluminescence reagent.

\section{Caspase- 3 activity assay}

The caspase- 3 assay was performed as described previously. ${ }^{23}$ According to the manufacturer's protocol, the activity of caspase-3 was evaluated by using a caspase-3 colorimetric assay kit (Abcam, Cambridge, UK). H460 and PC-9 cells were collected and resuspended in lysis buffer. After incubation on ice for $10 \mathrm{~min}$, the cell lysate was centrifuged at $12,000 \mathrm{rpm}$ for $10 \mathrm{~min}$ at $4{ }^{\circ} \mathrm{C}$, and the protein concentration in the supernatants was measured using the Bradford dye method. The supernatants were incubated with a reaction buffer containing $200 \mu \mathrm{M}$ devd-p-nitroanilide, for caspase- 3 in a caspase assay buffer at $37^{\circ} \mathrm{C}$ with $10 \mathrm{mM}$ dithiothreitol for $2 \mathrm{~h}$. The activity of caspase- 3 was determined by measuring the absorbance at $405 \mathrm{~nm}$. Each experiment was done in triplicate for three independent experiments.

STAT3 luciferase-report assay

STAT3 activation was detected by the STAT3 luciferase reporter plasmid (PGLSTAT3-LUC), as was previously described. ${ }^{23}$ Simply put, PC-9 and H460 cells were seeded in 24-well plates and incubated for $24 \mathrm{~h}$ before transfection. The cells were grown to different densities and co-transfected with pGLSTAT3-Luc and pRL-TK (a plasmid encoding Renilla luciferase) for $6 \mathrm{~h}$ using Lipofectamine 3000 (Invitrogen, Carlsbad, CA, USA). Finally, the cells were treated with different concentrations of gracillin for $12 \mathrm{~h}$. Luciferase activity was assessed by SpectraMax ID3 (Molecular Devices, San Jose, CA, USA). The inhibition of STAT3 activity by acetyl-bufalin was calculated as the ratio between the value of firefly and Renilla luciferase activity. Each experiment was done in triplicate in three independent experiments.
Proteome microarray assay and data analysis

Arrayit HuProt ${ }^{\mathrm{TM}}$ v2.0 $19 \mathrm{~K}$ Human Proteome Microarrays (CDI Laboratories, Baltimore, MD) were blocked for $1 \mathrm{~h}$ with $3 \% \mathrm{BSA}$ at room temperature. Biotinylated bufalin was diluted to $10 \mu \mathrm{M}$ in blocking buffer and cultured on the proteome microarray at room temperature for $1 \mathrm{~h}$. The arrays were washed with PBST (a mixture of PBS and Tween 20) and incubated with Cy3-Streptavidin at 1:1000 dilution (Sigma-Aldrich) for $1 \mathrm{~h}$ at room temperature. Finally, the microarray was spun dry and scanned with a GenePix 4200 A microarray scanner (Molecular Devices, San Jose, CA). Data were analysed by GenePix Pro 6.0 software. The signal-to-noise ratio (SNR) was defined as the ratio of median foreground value minus median background value. The cut-off value of SNR $(>1.1)$ was set.

Molecular docking of acetyl-bufalin to the binding spots of CDK9 Molecular docking was performed by using AutoDock Vina 1.0.21. ${ }^{24,25}$ The crystal structure of the human CDK9-A86 complex (PDB code 6GZH) derived from Protein Data Bank was used for our docking study. The input files of ligand and receptor were prepared using Graphical User Interface program AutoDock Tools 1.5.62 (The Scripps Research Institute, CA, USA). During the docking, the receptor was considered as rigid while the ligand was flexible.

\section{siRNA transfection}

The siRNA targeting human CDK9 was purchased from Genepharma (Shanghai, China) with the following sequence: siCDK9-1: 5'-GGCCAAACGUGGACAACUA Tा-3', siCDK9-2: 5'-GAAGGCUGC UAAUGUGCUUTT-3', siCDK9-3: 5'-CACUGGACCUCAUCGACAA-3'. Cells were transfected with $50 \mathrm{nM}$ siRNA using Lipofectamine 3000 (Invitrogen, CA, USA) for $48 \mathrm{~h}$.

\section{Co-immunoprecipitation assay}

In all, $1 \mathrm{mg}$ of whole-cell extracts were precleared with protein A/G agarose beads (Beyotime Institute of Biotechnology, China) for $2 \mathrm{~h}$ at $4{ }^{\circ} \mathrm{C}$. Immunoprecipitation was performed in the presence of the indicated primary antibody at $4{ }^{\circ} \mathrm{C}$ overnight. Immune complexes were captured by adding protein $A / G$ agarose beads and rotated at $4{ }^{\circ} \mathrm{C}$ for $2 \mathrm{~h}$. After the supernatant was discarded, protein $\mathrm{A} / \mathrm{G}$ agarose beads were washed with cold PBS for four to five times, and immunoprecipitates were fractionated by SDSPAGE.

\section{Animal model}

All animal procedures were done accordingly with the Institutional laboratory animal research guidelines and were approved by Wenzhou Medical University Animal Policy and Welfare Committee. Female athymic BALB/C nude mice (5-6 weeks old) and immune-defective non-obese or diabetic severe combined immunodeficiency (NOD-SCID) mice (6-8 weeks old) were purchased from the Vital River Experimental Animal Center (Beijing, China). All the mice were housed under $12 \mathrm{~h}$ light-dark cycles at $25^{\circ} \mathrm{C}$ and free for water and diet. For the xenograft model, the $\mathrm{H} 460$ cells $\left(5 \times 10^{6}\right.$ cells were mixed with an equal volume of PBS and Matrigel in $100 \mu \mathrm{L}$ ) were implanted on the hind flank of nude mice. Once tumour volumes reached $\sim 50 \mathrm{~mm}^{3}, 24$ mice were divided into four experimental groups (six mice per group, no differences in mean body weights or tumour volumes between the groups) and intraperitoneal injection with $2 \mathrm{mg} / \mathrm{kg}$ SNS-032 (a recognised CDK9 inhibitor), bufalin and acetyl-bufalin every other day. Tumour volume was measured as $\mathrm{V}=(\mathrm{L} \times \mathrm{W} \times$ $W) / 2$, in which $L$ and $W$ represent the length and width of tumour. Animals were killed by cervical dislocation once the tumour volume reached $1000 \mathrm{~mm}^{3}$. The tumours, hearts, livers, kidneys and lungs were removed for use in the histology and western blot analysis. 
For the patient-derived human NSCLC xenografts (PDXs) animal model, four pairs of lung cancer samples were obtained from patients who had undergone surgical treatment of lung cancer in the First Affiliated Hospital of Wenzhou Medical University. Fresh tumour tissue was sterilely incised into $\sim 3-\mathrm{mm}^{3}$ pieces and subcutaneously injected into immunodeficient NOD/SCID mice within $30 \mathrm{~min}$. The remaining tumour tissues were stored at $-80^{\circ}$ C. When the tumours have successfully engrafted, tumour samples were removed and passaged into the third generation of nude mice for the following studies. All 18 mice were allocated into three groups $(n=6)$ and received i.p. injection of acetyl-bufalin ( 1 $\mathrm{mg} / \mathrm{kg} / 2$ days and $2 \mathrm{mg} / \mathrm{kg} / 2$ days) as compared with mice injected with PBS (vehicle group) every other day. Tumour volume was measured as $V=(L \times W \times W) / 2$, in which $L$ and $W$ represent the length and width of tumour. After 14 days, all the mice were killed by cervical dislocation. The tumours, hearts, livers, kidneys and lungs were removed for use in the histology and western blot analysis.

\section{Immunohistochemistry staining}

Tumour tissues were fixed at room temperature in $10 \%$ paraformaldehyde and embedded in paraffin. Paraffinembedded tissue sections were $5 \mu \mathrm{m}$ thick. Then, the specimens were incubated overnight with anti-CDK9 antibody (Abcam, 1:100 dilution, in $1 \%$ bovine serum albumin PBS) at $4{ }^{\circ} \mathrm{C}$. The signal was detected by the corresponding secondary antibody. Thereafter, these slides were stained with diaminobenzidine and counterstained with haematoxylin. The images were captured using a light microscope.

Haematoxylin and eosin staining

The hearts, lungs, kidneys and livers of animals were fixed in $4 \%$ paraformaldehyde and embedded in paraffin. The paraffin tumour tissue sections $(5 \mu \mathrm{m})$ were deparaffinised and rehydrated and then stained with eosin and haematoxylin. The images were captured using a light microscope.

Statistical analysis

Data were expressed as mean \pm SD of three independent experiments. The statistical differences between different groups were analysed by the Student's $t$ test or one-way analysis of variance in GraphPad Pro7.0 (GraphPad, San Diego, CA). $P$ values less than $0.05(P<0.05)$ were considered indicative of significance.

\section{RESULTS}

Acetyl-bufalin changed the pharmacokinetic characteristics of bufalin and enhanced its efficacy

To solve the metabolic deficiency of bufalin and develop new analogues with higher efficiency and lower toxicity, the prodrug of bufalin, acetyl-bufalin was designed and synthesised (Fig. 1a and Supplementary Fig. S1). To investigate the effects of bufalin and acetyl-bufalin on human NSCLC cell lines, cell viability was evaluated via $\mathrm{MTT}$ assay. The $\mathrm{IC}_{50}$ values of bufalin and acetylbufalin were $140.9 \mathrm{nM}$ and $64.04 \mathrm{nM}$, respectively, in PC-9 cells (Fig. 1b), indicating that the inhibitory effect of acetyl-bufalin was approximately twice that of bufalin. Two human NSCLC cell lines, A549 and H460, were selected to observe the effect of acetylbufalin on NSCLC cell proliferation. The $\mathrm{IC}_{50}$ values were $28.11 \mathrm{nM}$ and $27.83 \mathrm{nM}$, respectively (Fig. 1b). Further, we choose two normal cells, human umbilical vein endothelial cell HUVEC and normal lung cells BEAS-2B, to analyse the cytotoxic effect of acetyl-bufalin with MTT assay. As shown in Supplementary Fig. S2a, acetyl-bufalin is low toxicity on normal cells compared with cancer cells. The effect of acetyl-bufalin on the cloning ability of NSCLC cells and normal cells was also explored. The colonyformation results showed that the NSCLC cell cloning ability was inhibited dose-dependently compared with normal cells (Fig. 1c and Supplementary Fig. S2b). To determine whether acetyl-bufalin could inhibit NSCLC cell migration, we performed a woundhealing assay using highly invasive PC-9 cells. Acetyl-bufalin markedly blocked PC-9 cell migration dose-dependently (Fig. 1d). Acute toxicity studies were performed to establish the safety of the compounds. The mice in the bufalin group (10/10) died immediately after the intraperitoneal injection of $30 \mathrm{mg} / \mathrm{kg}$ bufalin, while only $2 / 10$ mice died in the acetyl-bufalin group (Fig. 1e). Compared with the vehicle group, the weight of the remaining mice in the acetyl-bufalin group was stable (Fig. 1f). Then we established a highly sensitive and reliable LC-MS/MS method and analysed the rat plasma pharmacokinetics of bufalin and acetyl-bufalin after administration. After bufalin administrated to rats, bufalin showed fast absorption with the large maximum drug concentration ( $\mathrm{Cmax}$ ) and a short time to reach maximum drug concentration (tmax). While after acetyl-bufalin administrated to rats, the plasma concentrations of acetyl-bufalin and bufalin, which were produced by metabolism of acetyl-bufalin, were relatively lower with smaller $\mathrm{Cmax}$. However, the elimination of acetyl-bufalin and bufalin, which was produced by the metabolism of acetyl-bufalin in blood, was much slower than bufalin (Fig. 1g, h).

Acetyl-bufalin-induced apoptosis in human NSCLC cells To evaluate the apoptosis-inducing effects of acetyl-bufalin in human NSCLC cells, human NSCLC cells were treated with acetylbufalin for $24 \mathrm{~h}$, then stained with Annexin V-FITC and PI, and apoptotic cells were evaluated via flow cytometry. The data showed that acetyl-bufalin dose-dependently induced cell apoptosis in human NSCLC cells but did not affect normal human cells (Fig. 2a). Furthermore, the morphological features of apoptotic cells were visualised using Hoechst 33342 staining. Acetyl-bufalin was used to treat $\mathrm{H} 460$ and PC-9 cells for $24 \mathrm{~h}$; then the cells were stained with Hoechst 33342 . The percentage of acetyl-bufalininduced apoptotic cells was significant compared with the untreated vehicle group (Fig. 2b). In addition, western blot analysis and caspase-3 activity assay further revealed that acetylbufalin dose-dependently increased the expressions of the proapoptotic proteins, BAX and caspase- 3 activity, and decreased the level of the anti-apoptotic protein, BCL2, in human NSCLC cells (Fig. 2c, d and Supplementary Fig. S2c).

Acetyl-bufalin-induced G2/M cell cycle arrest and inhibited the STAT3 signalling pathway in human NSCLC cells

To assess the cell cycle arrest associated with acetyl-bufalin, PC-9 cell lines were treated with acetyl-bufalin for $12 \mathrm{~h}$, and the percentages of acetyl-bufalin-treated and untreated PC-9 cells in the G2/M phase were analysed via flow cytometry. Compared with the untreated vehicle group, acetyl-bufalin significantly induced the arrest of PC-9 cells in the G2/M phase of the cell cycle (Fig. 3a), followed by decreased cell growth and increased apoptosis. Western blot analysis indicated that treatment with acetyl-bufalin dose-dependently inhibited the expressions of cyclin B1 and CDC2 in human NSCLC cells (Fig. 3b and Supplementary Fig. S2d). Thus, the inhibitory effect of acetyl-bufalin on cell proliferation is partly associated with inducing G2/M phase arrest in human NSCLC cells.

A derivative of bufalin has been reported to play an anti-tumour role by inhibiting the STAT3 pathway; ${ }^{26}$ thus, we thought that acetyl-bufalin may affect STAT3 phosphorylation (P-STAT3). We tested the inhibitory ability of acetyl-bufalin on STAT3 phosphorylation in human NSCLC cells. STAT3 phosphorylation was assessed at different levels of confluence, as in the previous study. ${ }^{27}$ As expected, acetyl-bufalin reduced the P-STAT3 protein levels in H460, A549 and PC-9 cells concentration-dependently after $12 \mathrm{~h}$ of treatment. STAT3 participates in oncogenesis by mediating its target genes, including $B C L 2, M C L-1$, Survivin and VEGF. ${ }^{28}$ Analysis of the STAT3 target gene products revealed that acetyl-bufalin significantly reduced these target genes expression 


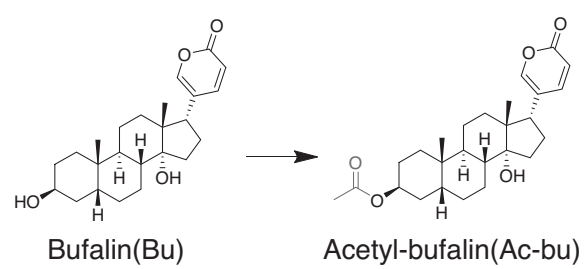

C
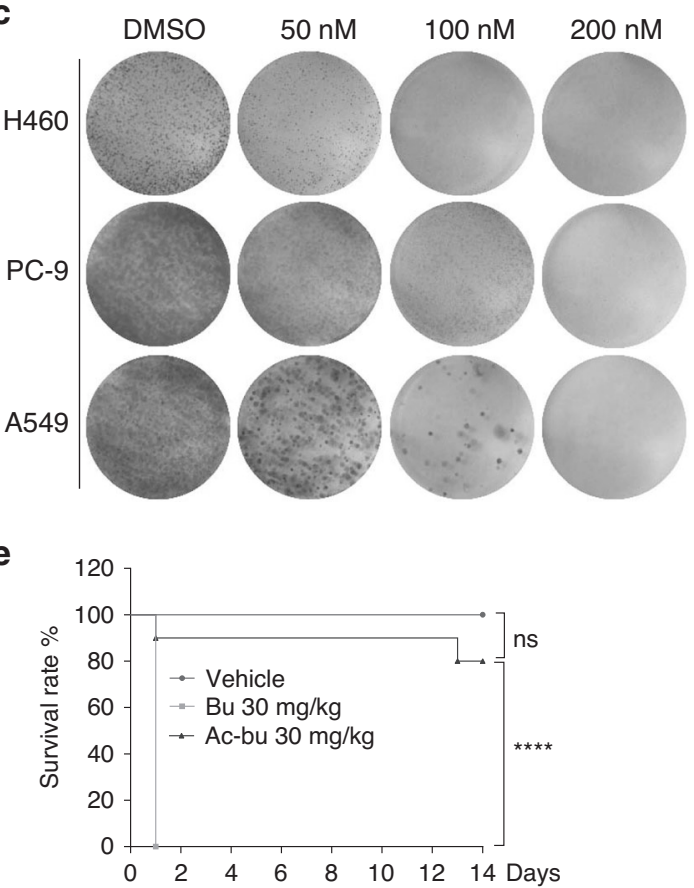

g

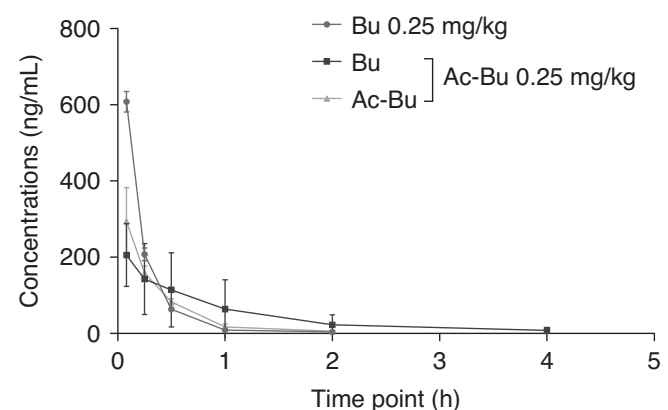

b

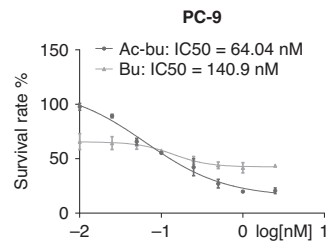

d

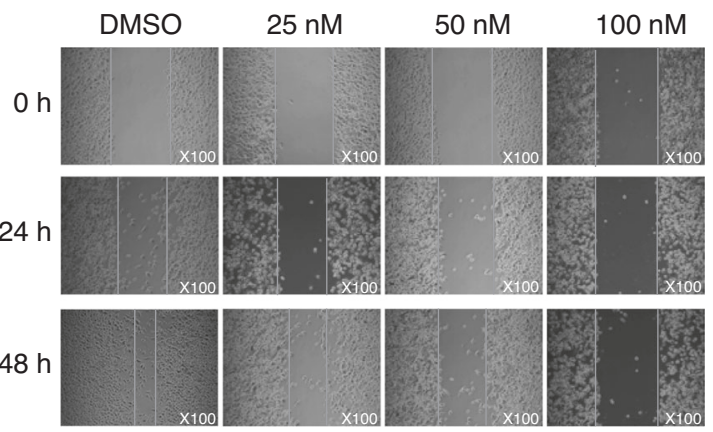

$\mathbf{f}$

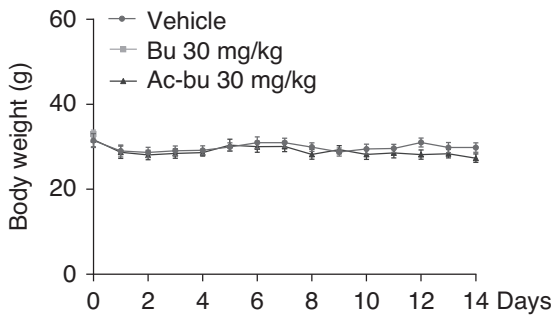

h

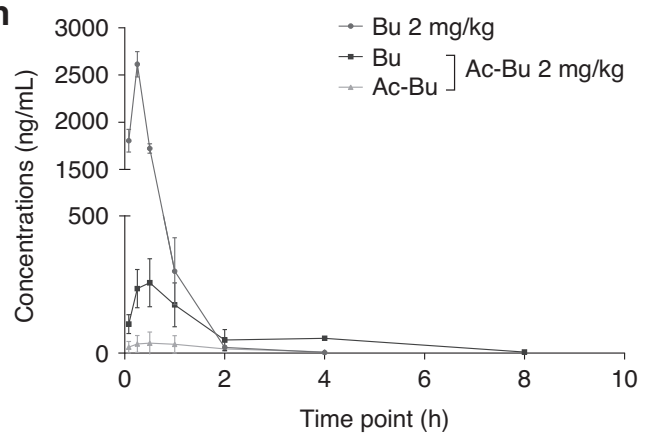

Fig. 1 Acetyl-bufalin changed the pharmacokinetic characteristics of bufalin and enhanced its efficacy. a Chemical structure of bufalin and acetyl-bufalin. b Human non-small-cell lung cancer (NSCLC) cells were incubated with increasing doses of bufalin (Bu) and acetyl-bufalin (Acbu) for $48 \mathrm{~h}$. Cell viability was determined via MTT assay, and IC $\mathrm{C}_{50}$ values were calculated. c Human NSCLC cells were incubated with acetylbufalin for $24 \mathrm{~h}$ and allowed to form colonies for 1 week. Colonies were then fixed, stained with crystal violet and photographed. $\mathbf{d}$ PC-9 cells were plated in six-well plates for $24 \mathrm{~h}$, then scratched and exposed to acetyl-bufalin for $48 \mathrm{~h}$ and observed microscopically at $\times 100$ magnification. e The bufalin and acetyl-bufalin groups received an intraperitoneal injection at the dose of $30 \mathrm{mg} / \mathrm{kg}$ on the first day, and the mortality and weight of the mice were observed for 14 days. ${ }^{* * *} P<0.0001$. $\mathbf{f}$ Body weight curve of the mice. $\mathbf{g}$ Mean concentration-time curves of bufalin and acetyl-bufalin in rat plasma after administration intravenously at a single dose of $0.25 \mathrm{mg} / \mathrm{kg}(n=3)$. $\mathbf{h}$ Mean concentration-time curves of bufalin and acetyl-bufalin in rat plasma after administration intraperitoneally at a single dose of $2 \mathrm{mg} / \mathrm{kg}(n=3)$.

(Fig. $3 c$ and Supplementary Fig. S3). In agreement with the data obtained using western blot assay, we found that acetyl-bufalin significantly inhibited P-STAT3 activation in a dose-dependent manner by using the luciferase reporter assay (Fig. 3d).

Proteomic identification of acetyl-bufalin-binding proteins and molecular docking

Based on previous studies, ${ }^{26}$ we speculated that STAT3 might be a direct target of acetyl-bufalin. To confirm our hypothesis, we screened for potential acetyl-bufalin-binding proteins. We first generated biotinylated bufalin (bio-bufalin; Fig. 4a and Supplementary Fig. S4). To ensure that the generated bio-bufalin retained its inhibitory activity in human NSCLC cells, we evaluated cell viability and compared the results with those of acetyl-bufalin. The $I C_{50}$ value of bio-bufalin in PC-9 cells was $77.07 \mathrm{nM}$, which was similar to the $\mathrm{IC}_{50}(64.20 \mathrm{nM})$ of acetyl-bufalin (Supplementary Fig. S5a). Therefore, bio-bufalin was selected for subsequent protein microarray study. We then used bio-bufalin in a human proteomic 
a

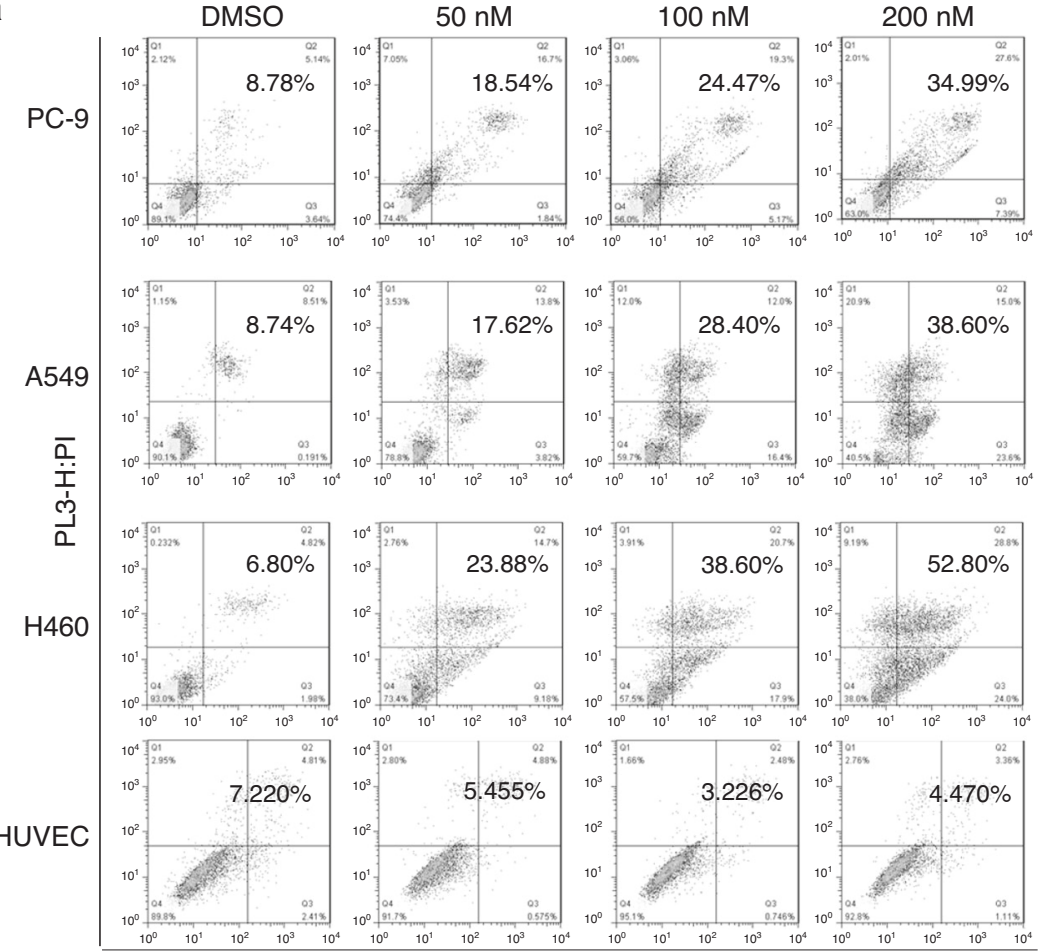

PL1-H:Amexin-V-FITC

b
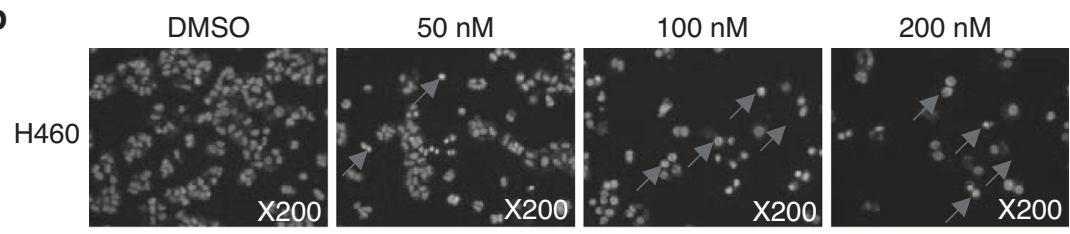

PC-9
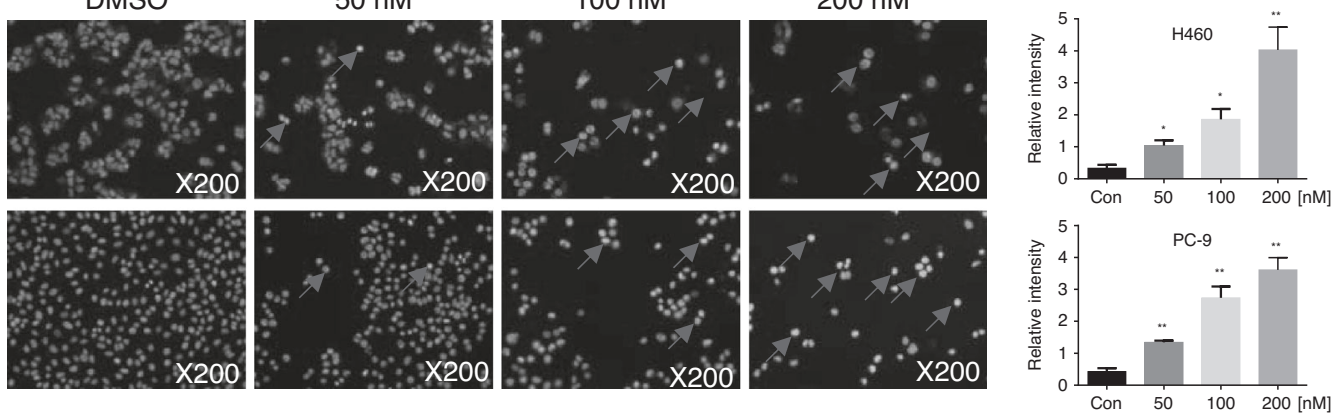

C

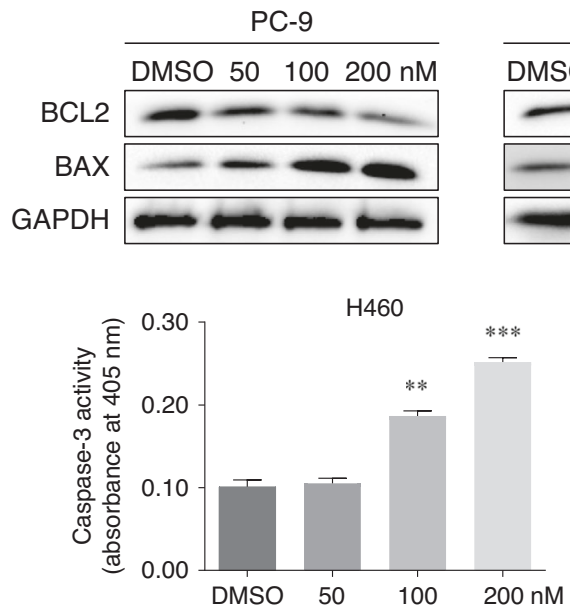

$\mathrm{H} 460$

DMSO $50100200 \mathrm{nM}$
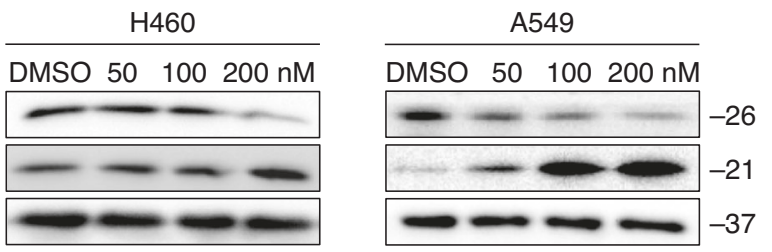

d

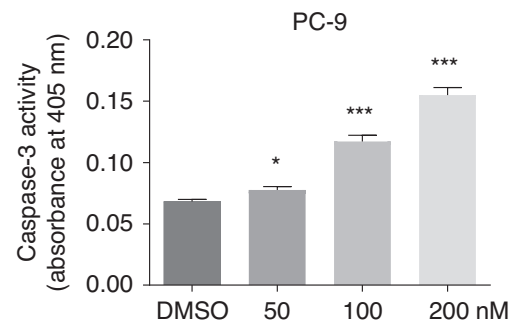

Fig. 2 Acetyl-bufalin-induced apoptosis in human non-small-cell lung cancer (NSCLC) cells. a Human H460, PC-9, A549 and HUVEC cells were treated with different concentrations of acetyl-bufalin for $24 \mathrm{~h}$, and the percentage of apoptotic cells was assessed using flow cytometry. Assays were performed in triplicate. b H460 and PC-9 cells were stained with Hoechst 33342 after $24 \mathrm{~h}$ of treatment with acetyl-bufalin and then observed under inverted fluorescence microscopy. c BCL2 and BAX expressions in H460, PC-9 and A549 cells were measured via western blot analysis after $24 \mathrm{~h}$ of treatment with acetyl-bufalin. d H460 and PC-9 cells were treated with different concentrations of acetyl-bufalin for $24 \mathrm{~h}$ then the activity of caspase-3 was determined. ${ }^{*} P<0.05,{ }^{* *} P<0.01,{ }^{* * *} P<0.001,{ }^{* * * *} P<0.0001$. 
a
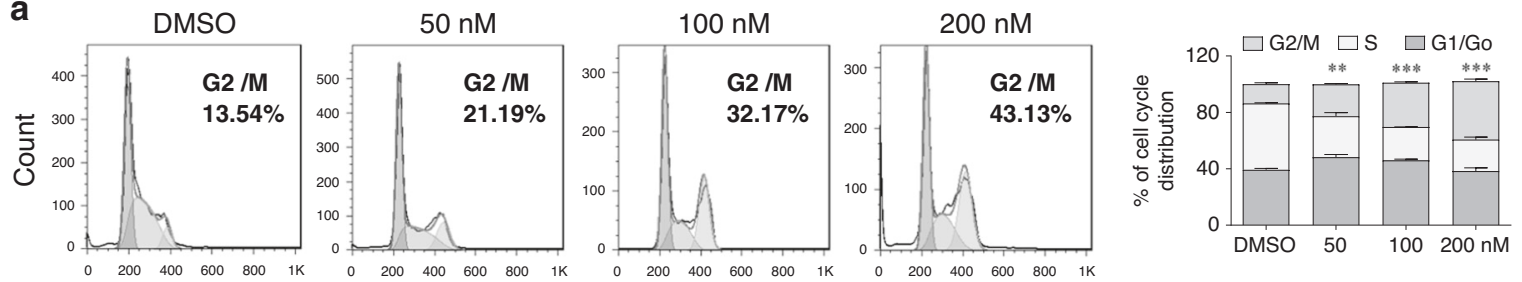

FL2-A

b

PC-9
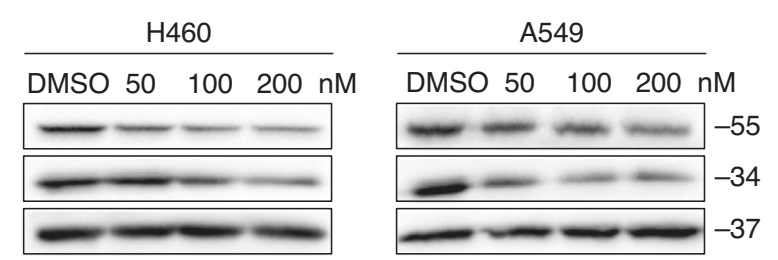

c

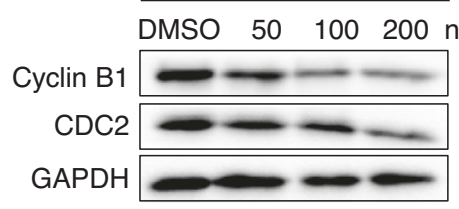

PC-9
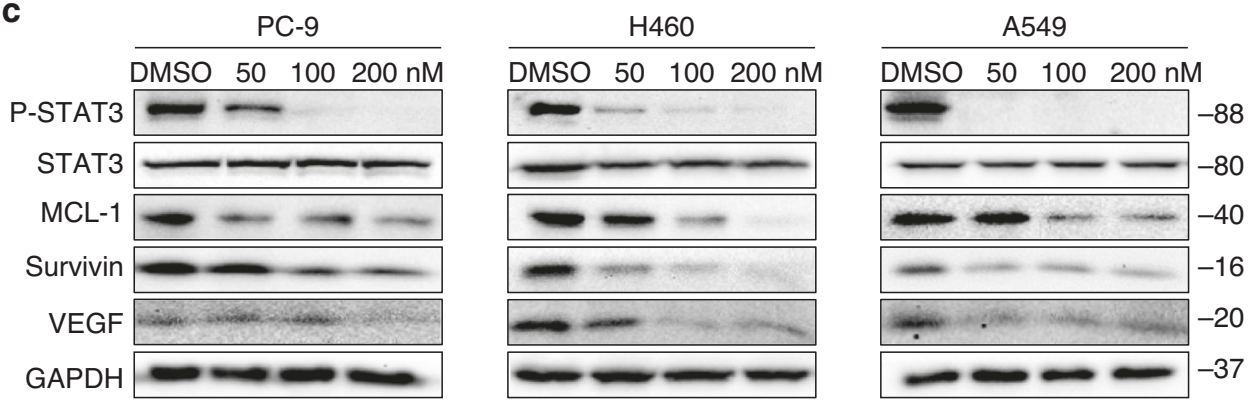

d
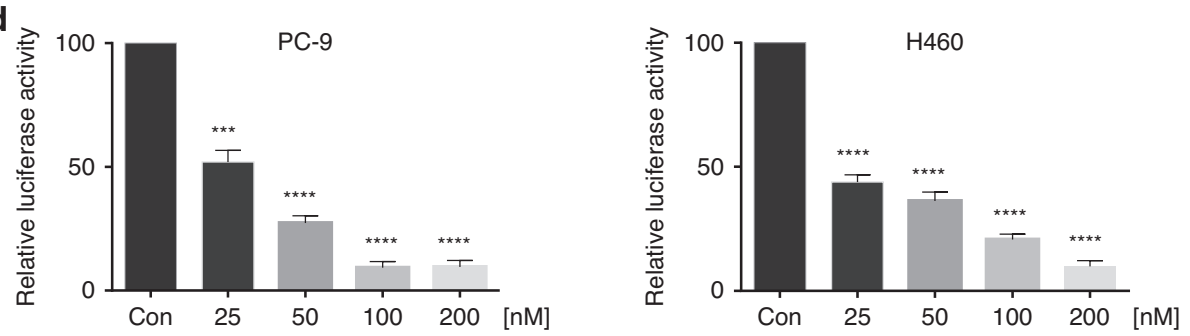

Fig. 3 Acetyl-bufalin induced G2/M cell cycle arrest and inhibited the STAT3 signalling pathway in human non-small-cell lung cancer (NSCLC) cells. a Cell cycle distribution in PC-9 cells exposed to acetyl-bufalin was assessed via flow cytometry. Histogram of cell cycle distribution in PC-9 cells with and without acetyl-bufalin treatment. b Human NSCLC cells were treated with four concentrations of acetylbufalin (0, 50, 100, $200 \mathrm{nM})$. Western blot analysis was used to detect the levels of cyclin B1 and CDC2 after acetyl-bufalin treatment. c H460, A549 and PC-9 cells were treated with concentration gradients of acetyl-bufalin. STAT3 and its downstream target genes, including MCL-1, Survivin and VEGF, were detected via western blot analysis. d PC-9 and H460 cells were transfected with luciferase reporter gene plasmid and treated with acetyl-bufalin $(0,25,50,100,200 \mathrm{nM})$ for $12 \mathrm{~h}$. The results were normalised to the Renilla luciferase activity. ${ }^{* *} P<0.01,{ }^{* * *} P<0.001$, $* * * * P<0.0001$.

microarray containing 19,394 affinity-purified N-terminal GSTtagged proteins covering $\sim 75 \%$ of the human proteome. Briefly, microarrays were probed with bio-bufalin, and binding was detected with a Cy3-conjugated streptavidin (Cy3-SA). We then calculated the SNR of each spot, which was defined as the ratio of the median foreground minus the median background. No STAT3 was found in 35 positive proteins with SNR values greater than 1.5, suggesting that bio-bufalin may not be a direct target of STAT3. However, CDK9 bound to bio-bufalin in 35 positive proteins, with the strongest SNR being 6.7478814 (Fig. 4b and Supplementary Fig. S5b). In addition, the docking result revealed that acetylbufalin occupied the ATP-binding site and formed three hydrogen bonds with residues ASP104, CYS106 and ASP109 (Fig. 4c). Thus, acetyl-bufalin may be an ATP-competitive inhibitor. Based on the HuProt ${ }^{\mathrm{TM}}$ human proteome microarray and docking results, we speculated that CDK9 may be a direct target of acetylbufalin.
Acetyl-bufalin inhibited the CDK9/STAT3 signalling pathway To investigate the CDK9 signalling pathway, we measured the expressions of several downstream target proteins of CDK9 after acetyl-bufalin treatment. After incubating $\mathrm{H} 460$ and PC-9 cells with $25,50,100$, or $200 \mathrm{nM}$ acetyl-bufalin and $200 \mathrm{nM}$ SNS-032 (a known CDK9 inhibitor) for $12 \mathrm{~h}, \mathrm{P}-\mathrm{CDK} 9$, CDK9, P-STAT3 and S2 RNAPII expressions were significantly dose-dependently decreased, whereas the total STAT3 and RNAPII expressions were not significantly changed (Fig. 4d and Supplementary Fig. S5c). IL6 is the main inducer of the STAT3 pathway in NSCLC and promotes tumour growth. CDK9 promoted IL6-induced STAT3 phosphorylation; conversely, CDK9 inhibition by siCDK9 blocked IL6-induced STAT3 phosphorylation dose-dependently (Fig. 4e, $\mathrm{f}$ and Supplementary Fig. S6a, b). To confirm the ability of acetyl-bufalin to reduce cell proliferation and test whether this effect was CDK9dependent, we examined the inhibitory effect of acetyl-bufalin on the proliferation of $\mathrm{H} 460$ and PC-9 cells depleted or not of CDK9. 
a

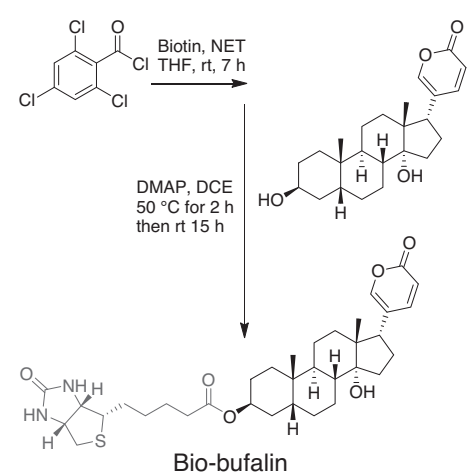

b

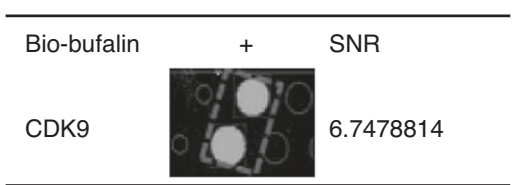

d

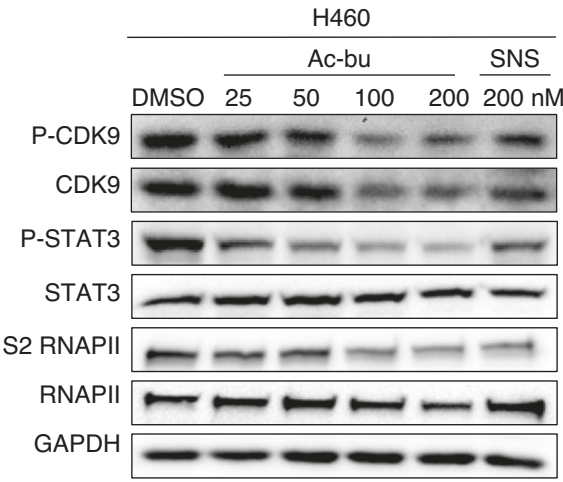

C

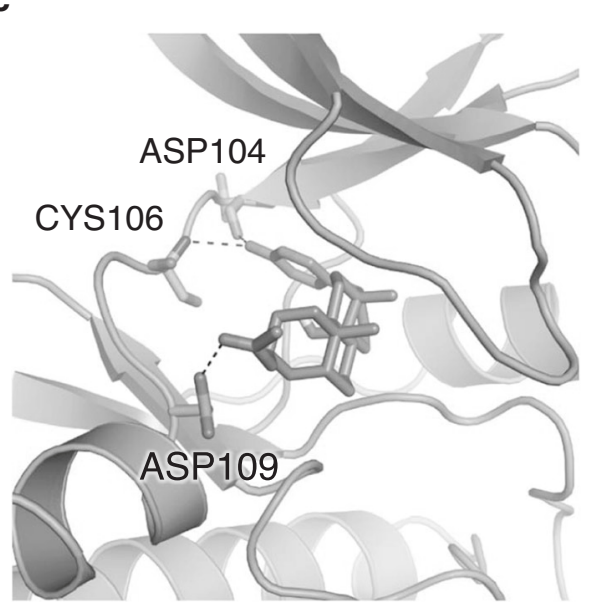

e

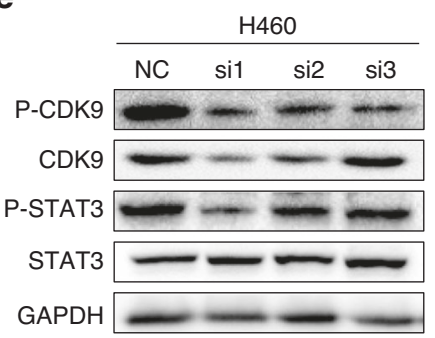

g

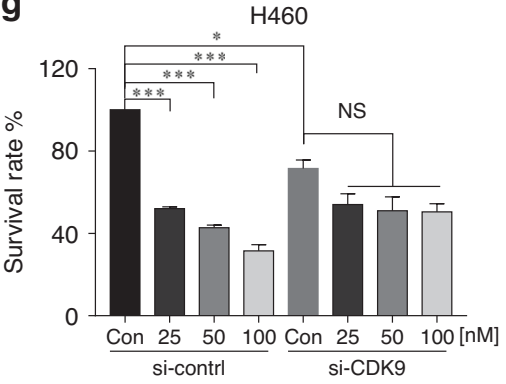

f
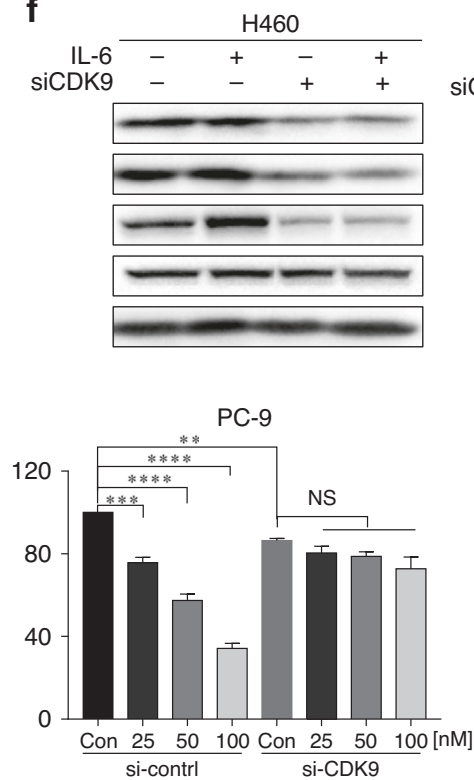

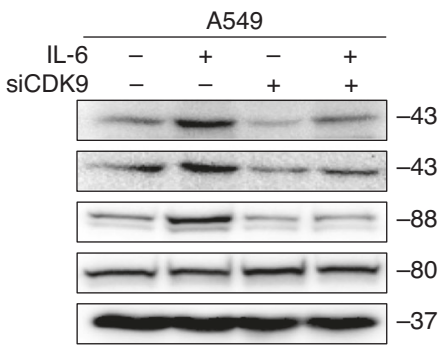

h

IB STAT3 -

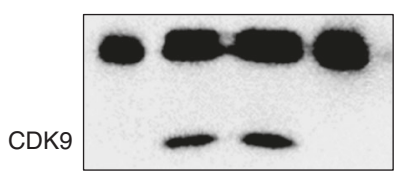

Fig. 4 Acetyl-bufalin inhibited the CDK9/STAT3 signalling pathway. a Chemical structure of biotin-labelled bufalin (bio-bufalin). b Magnified image of bio-bufalin binding to recombinant CDK9 protein spots on the microarray; signal-to-noise ratio (SNR) is shown. c Molecular docking of acetyl-bufalin to the CDK9 binding spots. d The expression of proteins involved in the CDK9/STAT3 pathway in H460 and PC-9 cells was examined by western blotting after $12 \mathrm{~h}$ of acetyl-bufalin (Ac-bu) and SNS-032 (SNS) treatment. e CDK9 and STAT3 expression levels by western blotting $48 \mathrm{~h}$ post transduction with CDK9 siRNA (si1, si2 and si3) in H460 cells. $\mathrm{f}$ Western blot analysis of CDK9 and STAT3 expression levels in H460 and PC-9 cells transfected with CDK9 siRNA1 and treated for 30 min with or without IL6 (25 ng/ml). The data are representative of three independent experiments. g Relative quantification of cell proliferation in $\mathrm{H} 460$ and PC-9 cells transfected with CDK9 siRNA1 and treated with a concentration gradient of acetyl-bufalin for $48 \mathrm{~h}$. $\mathbf{h}$ Co-immunoprecipitation analysis of STAT3 and CDK9 in H460 cells treated with IL6 $(25 \mathrm{ng} / \mathrm{ml}) .{ }^{*} P<0.05,{ }^{* *} P<0.01$, ${ }^{* * *} P<0.001,{ }^{* * * *} P<0.0001$. 


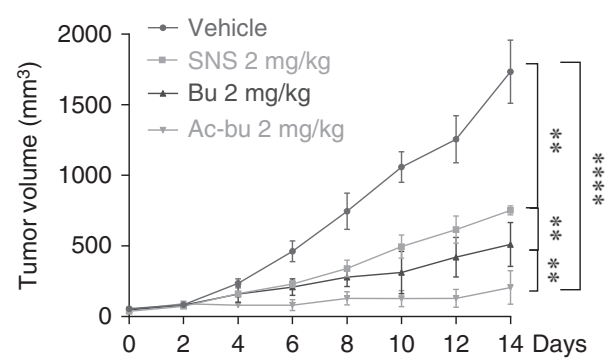

C

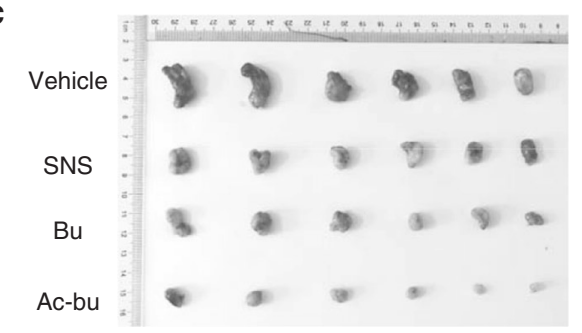

e

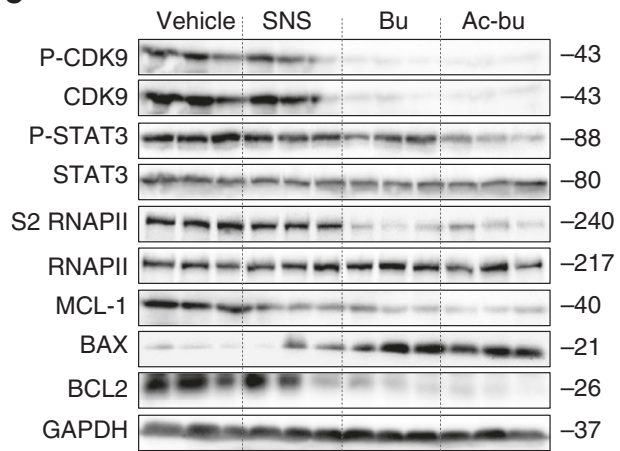

b

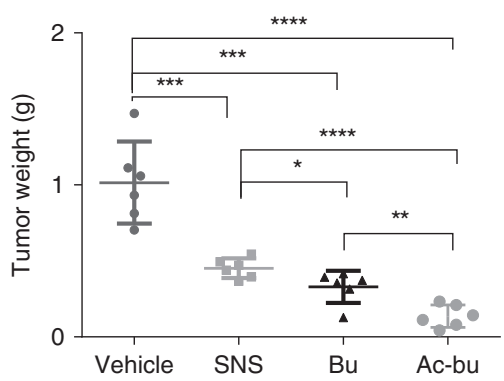

d

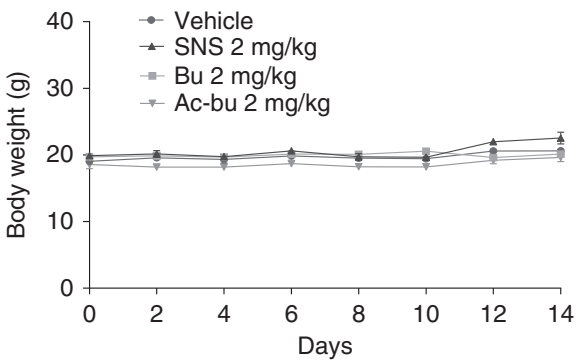

f

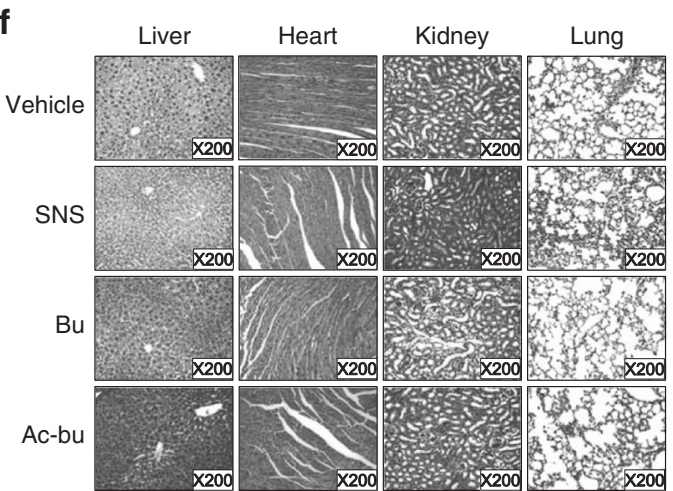

Fig. 5 Acetyl-bufalin inhibited growth of non-small-cell lung cancer (NSCLC) xenograft models. a, b Volume and weight of tumour tissue in anatomical nude mice treated with SNS-032(SNS), bufalin (Bu) or acetyl-bufalin (Ac-bu) $(2 \mathrm{mg} / \mathrm{kg})$. c Tumour tissue of nude mice treated with SNS-032(SNS), bufalin (Bu) or acetyl-bufalin (Ac-bu) $(2 \mathrm{mg} / \mathrm{kg})$. d Nude mouse size growth curve. e Western blot analysis on the expressions of CDK9/STAT3 pathway-associated proteins and apoptosis-related proteins in excised tumour tissue lysates. $f$ Haematoxylin and eosin staining of hearts, livers, lungs and kidneys from the nude mice at $\times 200$ magnification. ${ }^{*} P<0.05,{ }^{* *} P<0.01,{ }^{* * *} P<0.001,{ }^{* * *} P<0.0001$.

Acetyl-bufalin did not significantly affect the siCDK9 cells. Conversely, in cells transfected with a control siRNA (and therefore expressing (DK9), acetyl-bufalin hampered cell proliferation in H460 and PC-9 cells (Fig. 4g). These results strongly suggest that CDK9 is a direct target of acetyl-bufalin.

Next, we determined whether CDK9 interacted with STAT3 in human NSCLC cells, which was confirmed via coimmunoprecipitation experiments. H460 cells were stimulated with IL6 for $30 \mathrm{~min}$ or left untreated, whole-cell extracts were recovered, and coimmunoprecipitations were performed with polyclonal antibodies directed against CDK9, STAT3 or nonspecific antibodies. Under these conditions, CDK9 was coimmunoprecipitated with STAT3 proteins (Fig. 4h). These coimmunoprecipitations were carried out without using transfected cells so that the association did not require overexpressing the proteins. These results indicate that STAT3 interacts, likely directly, with CDK9.

Acetyl-bufalin inhibited NSCLC xenograft model growth Because of the potent cytotoxic activity of acetyl-bufalin on human NSCLC cells, we next assessed the therapeutic efficacy of acetyl-bufalin in $\mathrm{H} 460$ xenograft models. Mice were intraperitoneally injected with $2 \mathrm{mg} / \mathrm{kg}$ SNS-032, bufalin or acetyl-bufalin every other day. The tumour weight and volume were measured, and SNS-032, bufalin and acetyl-bufalin significantly inhibited tumour growth compared with that of the vehicle group. Acetylbufalin had the best inhibitory effect (Fig. 5a-c). Importantly, the body weights of the mice remained stable (Fig. $5 \mathrm{~d}$ ). Remarkably, PCDK9, P-STAT3 and S2 RNAPII expressions were decreased significantly after acetyl-bufalin treatment. In addition, acetylbufalin treatment markedly increased apoptosis as indicated by the BAX and BCL2 expressions (Fig. 5e) and had no gross toxicities on the hearts, livers, kidneys or lungs compared with those of the vehicle group (Fig. 5f), demonstrating excellent safety profiles.

Anti-tumour activity of acetyl-bufalin in a PDX animal model of NSCLC

PDX animal models may be superior to standard cell-line xenograft models of cancer because they are more similar to the parental tumours. PDX models are attractive choices for testing novel compounds. Four NSCLC specimens and their corresponding adjacent normal tissues were obtained surgically after primary diagnosis. To establish the PDX animal model, we designed a procedure, as shown in Fig. 6a. Of the four samples, only one passable xenograft model was successfully established. Third-generation mice were randomly divided into three groups. Tumour growth was obviously decreased in the acetyl-bufalintreated group (Fig. 6b). The average volumes of the tumours treated with acetyl-bufalin were $544.43 \mathrm{~mm}^{3}$ ( $1 \mathrm{mg} / \mathrm{kg}$ per 2 days) 


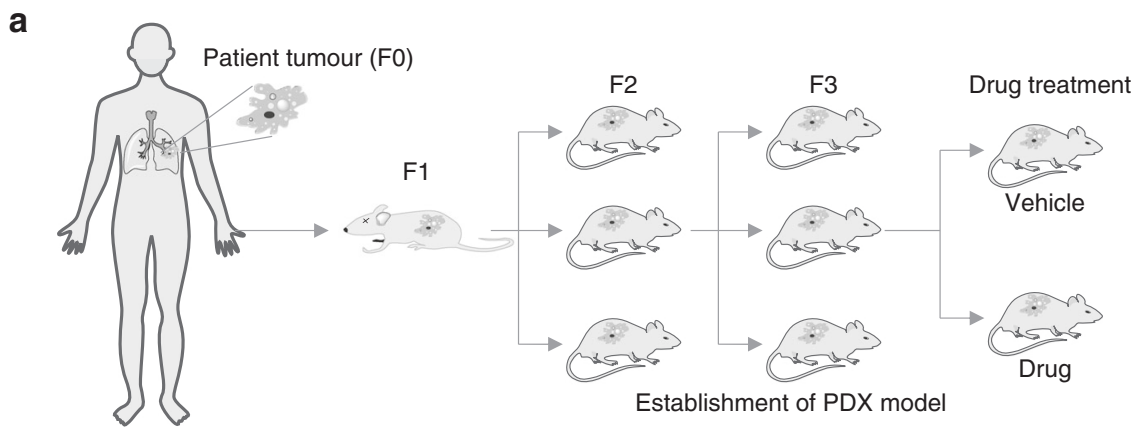

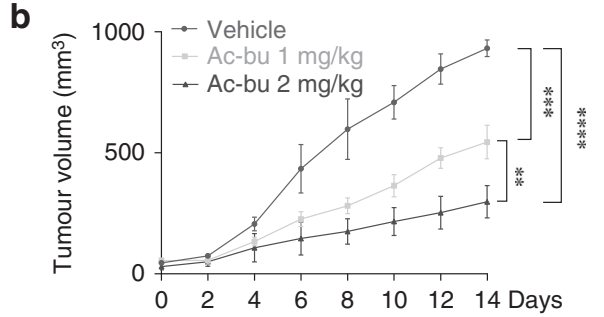

d

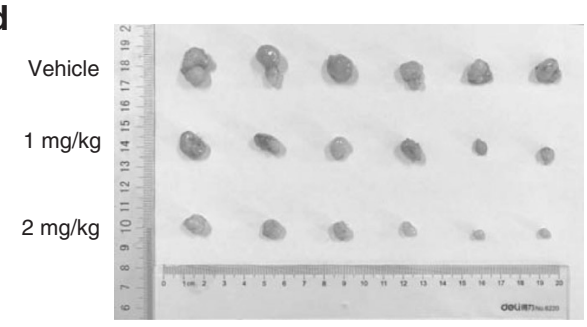

$\mathbf{f}$

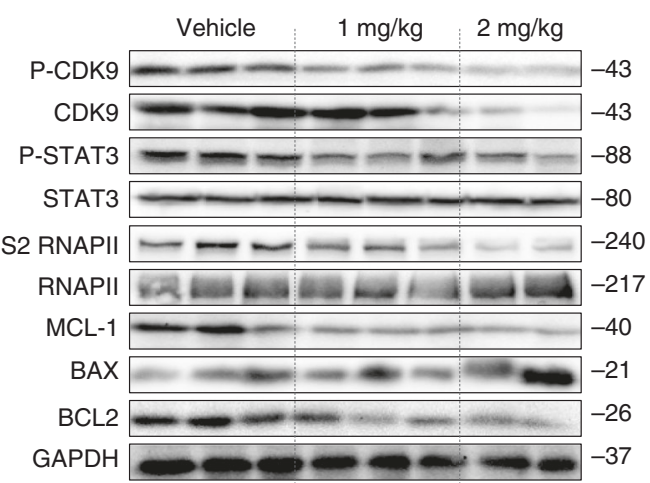

C

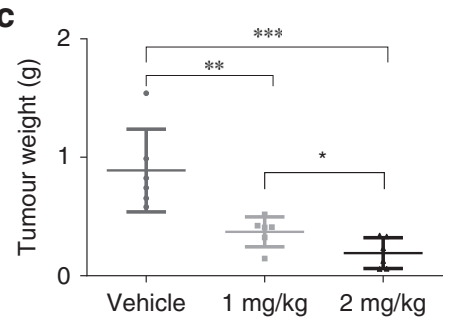

e

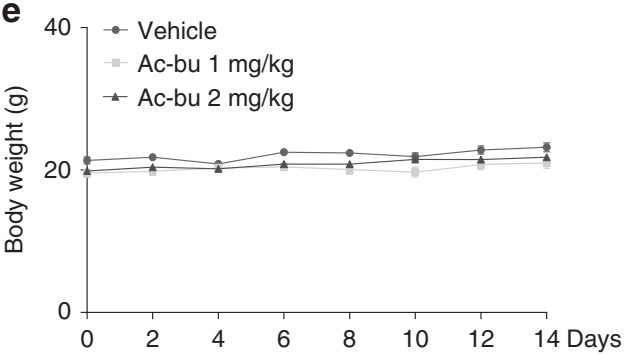

g

Vehicle $1 \mathrm{mg} / \mathrm{kg} \quad 2 \mathrm{mg} / \mathrm{kg}$

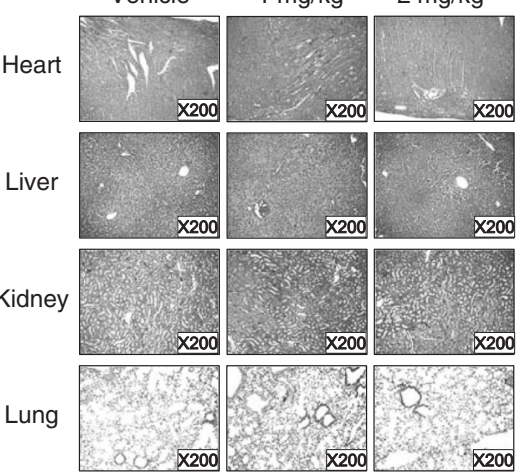

Fig. 6 Anti-tumour activity of acetyl-bufalin in a patient-derived (PDX) non-small-cell lung cancer (NSCLC) xenograft animal model. a Flowchart for establishing the PDX model. b Tumour volume in the PDX model. c Curve of the tumour weights in the mice. $\mathbf{d}$ Images of representative tumours removed from the PDX model. e Body weight curve of the PDX-model nude mice. $f$ Western blot analysis on the expressions of CDK9/STAT3 pathway-associated proteins and apoptosis-related proteins from tumour tissue removed from the PDX mouse model. g Haematoxylin and eosin staining of hearts, livers, lungs and kidneys from the nude mice at $\times 200$ magnification. ${ }^{*} P<0.05,{ }^{* *} P<0.01$, ***P $<0.001, * * * * P<0.0001$.

and $256.29 \mathrm{~mm}^{3}$ ( $2 \mathrm{mg} / \mathrm{kg}$ per 2 days). However, the average volume of the vehicle group tumours was $931.90 \mathrm{~mm}^{3}$ (Fig. 6b). Acetyl-bufalin led to a supra-additive reduction in growth in the xenograft models (Fig. 6c, d), and the body weight of the mice was stable (Fig. 6e). Notably, P-CDK9 and CDK9 expressions were downregulated after acetyl-bufalin treatment, indicating that CDK9 remained a major target of acetyl-bufalin in vivo (Fig. 6f). In addition, compared with the other three groups, acetyl-bufalin significantly decreased BCL2 levels and increased BAX levels. Moreover, the acetyl-bufalin treatment therapy had no gross toxicities on the heart, liver, kidney and lung compared with the vehicle group (Fig. 6g), demonstrating excellent safety profiles. These data indicated that the anti-tumour effects of acetyl-bufalin were due to inhibition of CDK9/STAT3 in the PDX animal model and supported that acetyl-bufalin may display potential therapeutic effects in clinical trials.

CDK9 is highly expressed in human NSCLC tissues

To assess the roles of CDK9 in human NSCLC, we first evaluated CDK9 protein expression in 12 human NSCLC tissues and their paired adjacent tissues. The CDK9 protein level was significantly upregulated in human NSCLC specimens compared with that of 
normal lung tissues. CDK9 was increased in 10 of the 12 tumour tissues (83.33\%) compared with that in the adjacent tissues (Supplementary Fig. S7a). To further confirm this result, we surveyed the CDK9 protein expression via immunohistochemistry. Immunohistochemistry analysis also showed that CDK9 was highly expressed in most human NSCLC tissues (Supplementary Fig. S7b). Collectively, our data suggest that CDK9 is significantly upregulated in NSCLC. Thus, CDK9 may serve as a novel diagnostic marker of NSCLC.

\section{DISCUSSION}

Enhanced circulation is the critical factor for promoting desired and acceptable pharmacokinetic behaviour leading to more delivery to the tumour site and less side effects. ${ }^{29,30}$ In vivo administration acetyl-bufalin led to the release of bufalin, which improved the pharmacokinetics of bufalin (Fig. 1g, h). Meanwhile, acetyl-bufalin was better tolerated in mice at a high-dose-level treatment than bufalin was (Fig. 1e, f). All these results demonstrated that acetyl-bufalin could change the pharmacokinetic characteristics of bufalin and prolong the action time, which means that it can reduce the times of administration and reduce the toxic and side effects caused by repeated bufalin administration. Instead, acetyl-bufalin has a safety profile that can be achieved by long intravenous infusions.

CDK9 inhibitors have been investigated as therapeutics for various haematological cancers and solid tumours. ${ }^{31-33}$ Several CDK9 inhibitors, such as AZD4573, BAY 1143572, FIT-039, THALSNS-032 and JSH-150, have been successfully developed and have shown promising efficacy in preclinical and clinical studies. ${ }^{19,32,34-36}$ To date, the activity observed does not match the initial expectations for CDK9 inhibitors, and no CDK9 inhibitor has been approved for clinical use, ${ }^{37,38}$ thus far, necessitating investigating more targeted approaches to improve outcomes. The optimal pharmacokinetic profile and dosing schedule for CDK9 inhibitors remain to be determined. ${ }^{20,37}$ In this study, acetyl-bufalin, a prodrug of bufalin, was designed and synthesised with higher efficiency and lower toxicity. Acetyl-bufalin can exert anti-tumour effect by inhibiting the CDK9/STAT3 signalling pathway (Fig. 4). Bufalin, as the main active constituent of chansu or Huachansu/cinobufacin, are already in use to treat patients, based on observed pharmacological activities, including anti-tumour, immunomodulation and attenuation of cancer-derived pain, ${ }^{11,39}$ but has adverse effects when administered as a single agent. ${ }^{12,14}$ Ideally, acetylbufalin is predicted to have a noncytotoxic mechanism of action towards noncancerous tissues (Figs. 5 and 6), and its pharmacokinetic profile should provide sufficient exposure throughout the whole-cell cycle with a reasonable administration schedule. However, the future anti-tumour research on acetyl-bufalin (and its analogues) needs to follow the standard pharmacology guidelines to ensure the efficacy and safety of chansu-derived substances based on evaluation of pharmacodynamic-related and toxicityrelated issues.

CDK9 is usually associated with transcriptional elongation, which can directly modulate translation through co-opting components of the major translational regulatory complex. ${ }^{18}$ Recent studies have identified other cellular factors that interact with CDK9, including mTOR, BRD4 and Hsp90. Two distinct complexes of CDK9 and mTOR (CTORC1 and CTORC2) play key roles in mRNA transcription and translation of mitogenic genes. ${ }^{40}$ Complex transcriptional elongation of BRD4 and CDK9 is necessary for TGF $\beta$-induced Nox4 expression and myofibroblast transdifferentiation. ${ }^{41}$ The Hsp90/Cdc37/CDK9 complex also exists in resting $\mathrm{T}$ cells lacking cyclin $\mathrm{T} 1 .{ }^{42}$ Sirtuin 2 (SIRT2)-mediated deacetylation of CDK9 deacetylation is essential for STAT1 phosphorylation at Ser-727. ${ }^{43}$ The STAT3-CDK9 complex can stimulate $\gamma$-FBG gene recruitment and prolong transcription. IL6 can increase both CDK9 and cyclin T1 protein levels in peripheral blood lymphocytes. ${ }^{21}$ STAT3 promotes transcription initiation and regulates chromatin remodelling and transcription elongation by interacting with BRG1 and CDK9. ${ }^{44}$ STAT3 phosphorylation occurs upon promoter binding by an unknown kinase. ${ }^{45,46}$ In our study, transient transfection assays revealed that CDK9 activity was required to activate IL6-induced STAT3, and western blotting showed that CDK9 knockdown significantly suppressed STAT3 expression. CDK9 was induced in response to IL6, which could be another mechanism for regulating STAT3 activity.

We demonstrate that the activity of CDK9 is necessary to maintain the transcription process induced by STAT3 proteins, which is activated abnormally by mutation and extracellular signal. MCL-1 and BCL2 are among the downstream genes transcriptionally regulated by the CDK9/STAT3 axis. It will be a significant research direction to determine whether CDK9 has a general mechanism to regulate some or all STAT3-dependent genes by detecting the kinetics of CDK9 association on different STAT3 target gene promoters through ChIP assays. Further studies of other STAT3 target genes may answer the question of whether all STAT3-dependent genes generally need CDK9 activity. Our findings that STAT3 phosphorylation is changed by CDK9 inhibition indicates that STAT3 may be a target of CDK9. Acetylbufalin reduces CDK9/RNA polymerase serine 2-mediated STAT3 transcription activity across representative NSCLC cell lines. Moreover, our data show that acetyl-bufalin treatment specifically and efficiently suppresses CDK9-STAT3 formation. This mechanism may be used to develop a novel therapeutic combination of acetyl-bufalin in NSCLC preclinical models. CDK9 is a novel prognostic marker and therapeutic target in ovarian cancer, osteosarcoma and leukaemia. ${ }^{16,36,47}$ Our findings also suggest that CDK9 may serve as a novel diagnostic marker of NSCLC.

\section{CONCLUSIONS}

It is conceivable that acetyl-bufalin can be further explored as a unique CDK9 inhibitor for anti-tumour therapy. Future studies will confirm the role of CDK9 in tumour metastasis and chemoresistance and explore the synergistic effects of acetyl-bufalin in combination with other chemotherapies.

\section{ACKNOWLEDGEMENTS}

We thank the Cardiothoracic Surgery Department of the First Affiliated Hospital of Wenzhou Medical University for kindly providing the tumour tissues.

\section{AUTHOR CONTRIBUTIONS}

L.H.Y., F.Z. and Y.Z. performed the research and drafted the paper; L.Y.X. and Y.N.L. collected clinical samples; H.Y.Z. and Y.Q.X. analysed the data; X.X.D. revised and edited the paper; Z.G.L. designed and synthesised acetyl-bufalin; X.Y.H., L.X.W. and C. G.Z. designed the research study; all authors have read and approved the final paper.

\section{ADDITIONAL INFORMATION}

Ethics approval and consent to participate All patients provided written informed consent to use their clinical data. The study was conducted in accordance with the national guidelines and acquired the approval of the Ethics Committee of the First Affiliated Hospital of Wenzhou Medical University. All animal procedures were done accordingly to the Institutional laboratory animal research guidelines and were approved by Wenzhou Medical University Animal Policy and Welfare Committee and were performed in accordance with the Declaration of Helsinki.

Consent to publish Not applicable.

Data availability All data and material presented in this article and in the Supplementary Information are available upon request from the corresponding author.

Competing interests The authors declare no competing interests. 
Funding information This work was financially supported by the National Natural Science Foundation of China (81902692 and 81973168), Natural Science Foundation of Zhejiang Province (LY20H160014 and LY19H160047), Medical Scientific Research Fund of Zhejiang Province (2019322308 and 2019RC204) and Wenzhou Municipal Science and Technology Bureau (ZY2020025, Y20190179 and Y20190056). Science and Technology Innovation Activity Plan for College Students of Zhejiang Province (2019R413083).

Supplementary information is available for this paper at https://doi.org/10.1038/ s41416-020-01135-6.

Note This work is published under the standard license to publish agreement. After 12 months the work will become freely available and the license terms will switch to a Creative Commons Attribution 4.0 International (CC BY 4.0).

Publisher's note Springer Nature remains neutral with regard to jurisdictional claims in published maps and institutional affiliations.

\section{REFERENCES}

1. Meng, Z., Garrett, C. R., Shen, Y., Liu, L., Yang, P., Huo, Y., Zhao, Q., Spelman, A. R. et al. Prospective randomised evaluation of traditional Chinese medicine combined with chemotherapy: a randomised phase II study of wild toad extract plus gemcitabine in patients with advanced pancreatic adenocarcinomas. Br. J. Cancer 107, 411-416 (2012)

2. Yang, T., Shi, R. L., Chang, L., Tang, K., Chen, K., Yu, G., Tian, Y. F., Guo, Y. L. et al. Huachansu suppresses human bladder cancer cell growth through the Fas/Fasl and TNF- alpha/TNFR1 pathway in vitro and in vivo. J. Exp. Clin. Cancer Res. 34, 21 (2015).

3. Ye, J., Chen, S. \& Maniatis, T. Cardiac glycosides are potent inhibitors of interferonbeta gene expression. Br. J. Cancer 7, 25-33 (2011).

4. Meng, Z., Yang, P., Shen, Y., Bei, W., Zhang, Y., Ge, Y., Newman, R. A., Cohen, L. et al. Pilot study of huachansu in patients with hepatocellular carcinoma, nonsmall-cell lung cancer, or pancreatic cancer. Cancer 115, 5309-5318 (2009).

5. Xiang, R. F., Wang, Y., Zhang, N., Xu, W. B., Cao, Y., Tong, J., Li, J. M., Wu, Y. L. et al. MK2206 enhances the cytocidal effects of bufalin in multiple myeloma by inhibiting the AKT/mTOR pathway. Cell Death Dis. 8, e2776 (2017).

6. Qi, F. H., Li, A. Y., Zhao, L., Xu, H. L., Inagaki, Y., Wang, D. L., Cui, X. Y., Gao, B. et al. Cinobufacini, an aqueous extract from Bufo bufo gargarizans Cantor, induces apoptosis through a mitochondria-mediated pathway in human hepatocellular carcinoma cells. J. Ethnopharmacol. 128, 654-661 (2010).

7. Zhan, X., Wu, H., Wu, H., Wang, R., Luo, C., Gao, B., Chen, Z. \& Li, Q. Natural active constituents of Bufo bufo gargarizans cantor: a review on pharmacological activity, toxicity and quality control. J. Ethnopharmacol. 246, 112178 (2020).

8. Wang, J. J., Zou, J. X., Xue, X. Q., Cai, D. M., Zhang, Y., Duan, Z. J., Xiang, Q. P., Yang, J. $C$. et al. ROR-gamma drives androgen receptor expression and represents a therapeutic target in castration-resistant prostate cancer. Nat. Med. 22, 488-496 (2016).

9. Prassas, I. \& Diamandis, E. P. Novel therapeutic applications of cardiac glycosides. Nat. Rev. Drug Discov. 7, 926-935 (2008).

10. Wang, Y., Lonard, D. M., Yu, Y., Chow, D. C., Palzkill, T. G., Wang, J., Qi, R. G., Matzuk, A. J. et al. Bufalin is a potent small-molecule inhibitor of the steroid receptor coactivators SRC-3 and SRC-1. Cancer Res. 74, 1506-1517 (2014).

11. Zhang, Y., Dong, Y., Melkus, M. W., Yin, S., Tang, S. N., Jiang, P., Pramanik, K., Wu, $\mathrm{W}$. et al. Role of P53-senescence induction in suppression of LNCaP prostate cancer growth by cardiotonic compound bufalin. Mol. Cancer Ther. 17, 2341-2352 (2018).

12. Yuan, Z., Yuan, Y., Han, L., Qiu, Y., Huang, X., Gao, F., Fan, G., Zhang, Y. et al. Bufalin-loaded vitamin $E$ succinate-grafted-chitosan oligosaccharide/RGD conjugated TPGS mixed micelles demonstrated improved antitumor activity against drug-resistant colon cancer. Int. J. Nanomed. 13, 7533-7548 (2018).

13. Yuan, J., Zeng, C., Cao, W., Zhou, X., Pan, Y., Xie, Y., Zhang, Y., Yang, Q. et al. Bufalin-loaded PEGylated liposomes: antitumor efficacy, acute toxicity, and tissue distribution. Nanoscale Res. Lett. 14, 223 (2019).

14. Chen, Q. \& Liu, J. Transferrin and folic acid co-modified bufalin-loaded nanoliposomes: preparation, characterization, and application in anticancer activity. Int. J. Nanomed. 13, 6009-6018 (2018).

15. Chai, X. P., Sun, G. L., Fang, Y. F., Hu, L. H., Liu, X. \& Zhang, X. W. Tumor-targeting efficacy of a BF211 prodrug through hydrolysis by fibroblast activation proteinalpha. Acta Pharm. Sin. 39, 415-424 (2018).

16. Ma, H. Z., Seebacher, N. A., Hornicek, F. J. \& Duan, Z. F. Cyclin-dependent kinase 9 (CDK9) is a novel prognostic marker and therapeutic target in osteosarcoma. Ebiomedicine 39, 182-193 (2019).

17. Wang, J. L., Dean, D. C., Hornicek, F. J., Shi, H. R. \& Duan, Z. F. Cyclin-dependent kinase 9 (CDK9) is a novel prognostic marker and therapeutic target in ovarian cancer. FASEB J. 33, 5990-6000 (2019).
18. Brauns-Schubert, P., Schubert, F., Wissler, M., Weiss, M., Schlicher, L., Bessler, S., Safavi, M., Miething, C. et al. CDK9-mediated phosphorylation controls the interaction of TIP60 with the transcriptional machinery. EMBO Rep. 19, 244-256 (2018).

19. Parua, P. K., Booth, G. T., Sanso, M., Benjamin, B., Tanny, J. C., Lis, J. T. \& Fisher, R. P. A Cdk9-PP1 switch regulates the elongation-termination transition of RNA polymerase II. Nature 558, 460-464 (2018).

20. Olson, C. M., Jiang, B. S., Erb, M. A., Liang, Y. K., Doctor, Z. M., Zhang, Z. N., Zhang, T. H., Kwiatkowski, N. et al. Pharmacological perturbation of CDK9 using selective CDK9 inhibition or degradation. Nat. Chem. Biol. 14, 163 (2018).

21. Hou, T., Ray, S. \& Brasier, A. R. The functional role of an interleukin 6-inducible CDK9 center dot STAT3 complex in human gamma-fibrinogen gene expression. J. Biol. Chem. 282, 37091-37102 (2007).

22. Yang, L., Li, J., Xu, L., Lin, S., Xiang, Y., Dai, X., Liang, G., Huang, X. et al. Rhein shows potent efficacy against non-small-cell lung cancer through inhibiting the STAT3 pathway. Cancer Manag. Res. 11, 1167-1176 (2019).

23. Yang, L., Lin, S., Kang, Y., Xiang, Y., Xu, L., Li, J., Dai, X., Liang, G. et al. Rhein sensitizes human pancreatic cancer cells to EGFR inhibitors by inhibiting STAT3 pathway. J. Exp. Clin. Cancer Res. 38, 31 (2019).

24. Trott, O. \& Olson, A. J. Software news and update autodock vina: improving the speed and accuracy of docking with a new scoring function, efficient optimization, and multithreading. J. Comput. Chem. 31, 455-461 (2010).

25. Morris, G. M., Huey, R., Lindstrom, W., Sanner, M. F., Belew, R. K., Goodsell, D. S. \& Olson, A. J. AutoDock4 and AutoDockTools4: automated docking with selective receptor flexibility. J. Comput. Chem. 30, 2785-2791 (2009).

26. Wu, X. Y., Tian, F., Su, M. H., Wu, M., Huang, Y., Hu, L. H., Jin, L. \& Zhu, X. J. BF211, a derivative of bufalin, enhances the cytocidal effects in multiple myeloma cells by inhibiting the IL-6/JAK2/STAT3 pathway. Int. Immunopharmacol. 64, 24-32 (2018).

27. Vultur, A., Arulanandam, R., Turkson, J., Niu, G., Jove, R. \& Raptis, L. Stat3 is required for full neoplastic transformation by the Simian Virus 40 large tumor antigen. Mol. Biol. Cell 16, 3832-3846 (2005).

28. Zhang, T., Li, J., Yin, F., Lin, B., Wang, Z., Xu, J., Wang, H., Zuo, D. et al. Toosendanin demonstrates promising antitumor efficacy in osteosarcoma by targeting STAT3. Oncogene 36, 6627-6639 (2017).

29. Ekladious, I., Colson, Y. L. \& Grinstaff, M. W. Polymer-drug conjugate therapeutics: advances, insights and prospects. Nat. Rev. Drug Discov. 18, 273-294 (2019).

30. Xiao, M., Lai, W., Wang, F., Li, L., Fan, C. \& Pei, H. Programming drug delivery kinetics for active burst release with DNA toehold switches. J. Am. Chem. Soc. 141, 20354-20364 (2019).

31. Boffo, S., Damato, A., Alfano, L. \& Giordano, A. CDK9 inhibitors in acute myeloid leukemia. J. Exp. Clin. Cancer Res. 37, 1-10 (2018).

32. Alcon, C., Manzano-Munoz, A. \& Montero, J. A new CDK9 inhibitor on the block to treat hematological malignancies. Clin. Cancer Res. 26, 761-763 (2019)

33. Phillips, D. C., Jin, S., Gregory, G. P., Zhang, Q., Xue, J., Zhao, X., Chen, J., Tong, Y. et al. A novel CDK9 inhibitor increases the efficacy of venetoclax (ABT-199) in multiple models of hematologic malignancies. Leukemia 34, 1646-1657 (2019).

34. Ajiro, M., Sakai, H., Onogi, H., Yamamoto, M., Sumi, E., Sawada, T., Nomura, T., Kabashima, K. et al. CDK9 inhibitor FIT-039 suppresses viral oncogenes E6 and E7 and Has a therapeutic effect on HPV-induced neoplasia. Clin. Cancer Res. 24, 4518-4528 (2018).

35. Kinoshita, S., Ishida, T., Ito, A., Narita, T., Masaki, A., Suzuki, S., Yoshida, T., Ri, M. et al. Cyclin-dependent kinase 9 as a potential specific molecular target in NK-cell leukemia/lymphoma. Haematologica 103, 2059-2068 (2018).

36. Narita, T., Ishida, T., Ito, A., Masaki, A., Kinoshita, S., Suzuki, S., Takino, H., Yoshida, T. et al. Cyclin-dependent kinase 9 is a novel specific molecular target in adult Tcell leukemia/lymphoma. Blood 130, 1114-1124 (2017).

37. Bragelmann, J., Dammert, M. A., Dietlein, F., Heuckmann, J. M., Choidas, A., Bohm, S., Richters, A., Basu, D. et al. Systematic kinase inhibitor profiling identifies CDK9 as a synthetic lethal target in NUT midline carcinoma. Cell Rep. 20, 2833-2845 (2017).

38. Wang, B. L., Wu, J. X., Wu, Y., Chen, C., Zou, F. M., Wang, A. L., Wu, H., Hu, Z. Q. et al. Discovery of 4-(((4-(5-chloro-2-(((1s,4s)-4-((2-methoxyethyl)amino) cyclohexyl) amino)pyridin-4-yl)thiazol-2-yl)amino)methyl) tetrahydro-2H-pyran-4-carbonitrile (JSH-150) as a novel highly selective and potent CDK9 kinase inhibitor. Eur. J. Med. Chem. 158, 896-916 (2018).

39. Sun, X., Ng, T. T. H., Sham, K. W. Y., Zhang, L., Chan, M. T. V., Wu, W. K. K. \& Cheng, C. H. K. Bufalin, a traditional Chinese medicine compound, prevents tumor formation in two murine models of colorectal cancer. Cancer Prev. Res. 12, 653-666 (2019).

40. Beauchamp, E. M., Abedin, S. M., Radecki, S. G., Fischietti, M., Arslan, A. D., Blyth, G. T., Yang, A., Lantz, C. et al. Identification and targeting of novel CDK9 complexes in acute myeloid leukemia. Blood 133, 1171-1185 (2019).

41. Ijaz, T., Jamaluddin, M., Zhao, Y. X., Zhang, Y. Q., Jay, J., Finnerty, C. C., Herndon, D. N., Tilton, R. G. et al. Coordinate activities of BRD4 and CDK9 in the transcriptional elongation complex are required for TGF beta-induced Nox4 expression and myofibroblast transdifferentiation. Cell Death Dis. 8, e2606 (2017). 
42. Mbonye, U., Wang, B. L., Gokulrangan, G., Shi, W. X., Yang, S. C. \& Karn, J. Cyclindependent kinase 7 (CDK7)-mediated phosphorylation of the CDK9 activation loop promotes P-TEFb assembly with Tat and proviral HIV reactivation. J. Biol. Chem. 293, 10009-10025 (2018).

43. Kosciuczuk, E. M., Mehrotra, S., Saleiro, D., Kroczynska, B., Majchrzak-Kita, B., Lisowski, P., Driehaus, C., Rogalska, A. et al. Sirtuin 2-mediated deacetylation of cyclin-dependent kinase 9 promotes STAT1 signaling in type I interferon responses. J. Biol. Chem. 294, 827-837 (2019).

44. Giraud, S., Hurlstone, A., Avril, S. \& Coqueret, O. Implication of BRG 1 and cdk9 in the STAT3-mediated activation of the p21waf1 gene. Oncogene 23, 7391-7398 (2004).
45. Zhao, C. G., Li, H. M., Lin, H. J., Yang, S. L., Lin, J. Y. \& Liang, G. Feedback activation of STAT3 as a cancer drug-resistance mechanism. Trends Pharm. Sci. 37, 47-61 (2016).

46. Yang, L., Lin, S., Xu, L., Lin, J., Zhao, C. \& Huang, X. Novel activators and smallmolecule inhibitors of STAT3 in cancer. Cytokine Growth Factor Rev. 49, 10-22 (2019).

47. Minzel, W., Venkatachalam, A., Fink, A., Hung, E., Brachya, G., Burstain, I., Shaham, M. Rivlin, A. et al. Small molecules co-targeting CKI alpha and the transcriptional kinases $C D K 7 / 9$ control $A M L$ in preclinical models. Cell 175, 171-185 (2018). 